\title{
A non-linear homogeneous model for bone-like materials under compressive load.
}

\author{
M. Mengoni ${ }^{\star}$, R. Voide ${ }^{\star \star}$, D. Toye ${ }^{\star \star \star}$, A. Léonard ${ }^{\star \star \star}$, G.H. van Lenthe ${ }^{\star \star}$ and J.P. Ponthot ${ }^{\star}$ \\ ${ }^{\star}$ University of Liège, LTAS - Non Linear Computational Mechanics, Liège, Belgium, \\ mmengoni@ulg.ac.be \\ ${ }^{\star \star}$ ETH Zurich, Institute for Biomechanics, Zurich, Switzerland \\ ${ }^{\star \star \star}$ University of Liège, Department of Applied Chemistry, Laboratory of Chemical Engineering, \\ Liège, Belgium
}

\begin{abstract}
SUMMARY
Using morphological data provided by computed tomography, finite element (FE) models can be used to compute the mechanical response of bone and bone-like materials without describing the complex local microarchitecture. A constitutive law is here developed and proposed for this purpose. It captures the non-linear structural behavior of bone-like materials through the use of fabric tensors. It also allows for irreversible strains using a plastic material model, allowing hardening of the yield parameters. These characteristics are expressed in a constitutive law based on the anisotropic continuum damage theory coupled with isotropic elastoplasticity in a finite strains framework. This law is implemented into Metafor, a non-linear FE software. Simulations of cylindrical samples undergoing stepwise compression are presented.
\end{abstract}

Key Words: trabecular bone, constitutive law, plasticity, anisotropy, fabric.

\section{INTRODUCTION}

Within the diverse approaches that have been adopted to model trabecular bone remodeling processes, most of them are qualified as phenomenological models. They are models whose goal is to predict the global mechanical behavior (displacement, strains and stresses) of a tissue or an organ, taking into account the applied loads, its microstructure and the constraints imposed at the boundaries. Most of these models assume the existence of a given mechanical stimulus (input) that produces bone apposition or resorption (output) in such a way that the stimulus tends to a physiological level in the long-term (homeostasis). These phenomenological models are therefore build on constitutive material laws linking non-local stresses to global strains with internal variables representative of the evolving local microstructure. These models therefore need not only to be validated against remodeling data but the global constitutive law itself needs to be validated as well.

The goal of this work is therefore to present a non-local constitutive law that can be used in bone remodeling simulations. The constitutive law therefore aims at describing the mechanical behavior of trabecular bone in the range of small to moderate compressive strains, considering accumulation of plastic deformation and possible low softening due to small buckling of the microstructure. Under compressive load, the mechanical behavior of trabecular bone shows characteristics of an elastoplastic cellular solid, hence, the proposed global mechanical behavior is to be validated against experimental data obtained for compressive tests of trabecular bone as well as materials with bone-like microstructure. 


\section{METHODS}

The original remodeling law which is proposed is built on a damage/repair model, first proposed by Doblaré and co-workers [1]. It is enhanced to be coupled to an elastoviscoplastic material behavior in a finite strains framework. It can therefore capture permanent strains of the tissue beyond the ones due to the density variation. However, we present here only the purely mechanical part of the constitutive law, no damage variation due to remodeling is accounted for.

The bone tissue (global level) is considered as an anisotropic "organization" of elastoplastic trabeculae (local level). This "organization", as proposed in [1], is measured through a mean bone density and its anisotropy uses the concept of fabric tensor [2]. The continuum damage framework is used not to capture actual damage at the local level, i.e. micro-cracks of the trabeculae, but to represent the bone macroscopic porosity and therefore measure the volume fraction. In terms of morphological data provided by computed tomography, damage is therefore to be understood as a measure of the apparent density of the tissue $(\bar{\rho})$ while the anisotropy is measured by the fabric tensor (the fabric tensor $\hat{\boldsymbol{H}}$ is normalized in such a way that $\operatorname{tr}(\hat{\boldsymbol{H}})=3$ ). The undamaged material is therefore the ideal virtual situation of bone with null porosity and perfect isotropy. We use a tensor damage variable, $\boldsymbol{d}$, formulated so that it retrieves an isotropic definition as $d=1-E / E_{0}$, $E$ being the Young's modulus of the bone tissue and $E_{0}$ the Young's modulus of the trabeculae and depends on the morphological parameters :

$$
\boldsymbol{d}=\boldsymbol{I}-\bar{\rho}^{\beta} A \hat{\boldsymbol{H}}
$$

where $A$ is a calibration parameter to retrieve the damage definition in isotropy and $\beta$ is defined so that the tissue young's modulus is related to the density as : $E(\rho) \propto \rho^{\beta}$

For an elasto-plastic material in an hypoelastic formulation, the basic assumption consists in an additive split of the strain rate into two parts : an elastic and reversible part $\boldsymbol{D}^{e}$ and an irreversible plastic part $\boldsymbol{D}^{p}$. The constitutive model defines an effective stress, $\tilde{\boldsymbol{\sigma}}$, acting on the effective area of the material. The effective stress rate is linked to the elastic strain rate by the generalized Hooke's law :

$$
\dot{\tilde{\boldsymbol{\sigma}}}=\mathbb{C}:\left(\boldsymbol{D}-\boldsymbol{D}^{p}\right)
$$

where $\mathbb{C}$ is the elastic stiffness tensor. The stress $(\boldsymbol{\sigma}=\boldsymbol{s}+p \boldsymbol{I})$ is obtained by taking into consideration the effect of the structural morphology represented by the damage tensor [3] :

$$
\tilde{\boldsymbol{\sigma}}=\operatorname{dev}(\boldsymbol{H} \boldsymbol{s} \boldsymbol{H})+\frac{p}{1-\frac{\eta}{3} d_{k k}} \boldsymbol{I}
$$

where, $\boldsymbol{H}=(\boldsymbol{I}-\boldsymbol{d})^{-1 / 2}$ and $\eta$ is the degree of anisotropy. This reduces, when an isotropic fabric is considered, as $\tilde{\boldsymbol{\sigma}}=\boldsymbol{\sigma} /(1-d)$.

The plastic part of the strain rate $D^{p}$ can be calculated through the normality rule on the plastic criterion (associated plasticity) expressed in term of effective stress. This is therefore the plastic criterion one would get from a single trabeculea mechanical behavior. It is here assumed to be pressure independent and therefore a simple Von-Mises criterion is chosen, leading, when only isotropic hardening of the internal variable is allowed, to :

$$
\boldsymbol{D}^{p l}=\frac{3}{2} \frac{\dot{\lambda}}{\tilde{\sigma}_{e q}} \operatorname{dev}(\boldsymbol{H} \tilde{\boldsymbol{s}} \boldsymbol{H})
$$

where $\dot{\lambda}$ is the rate of plastic multiplier and $\tilde{\sigma}_{e q}$ is the equivalent stress used for the Von-Mises criterion.

The constitutive law is integrated in a finite element framework according to an iterative staggered scheme [4] and is implemented in Metafor [5], a in-house object-oriented finite element code. 


\section{RESULTS}

This homogeneous formulation has been applied to several materials, all showing bone-like microstructure. For each type of material, cylindrical specimens were used. Images of the structure were acquired using a micro-tomographic imaging system. These images were analyzed to extract the morphological data needed to compute the parameters of the presented constitutive law. The samples were also compressed along their main axis in a mechanical testing device and load/displacement data were acquired.

The first material that has been tested is a porous aluminium alloy (Duocel 6101-T6, ERG, Oakland, CA). The specimen used ( $8 \mathrm{~mm}$ in diameter and $16 \mathrm{~mm}$ in height, with an apparent volume density of $11.8 \%$ ) was arbitrarily chosen among 15 compressed and imaged specimens [6]. The compression was realized in a stepwise fashion from $0 \%$ to $16 \%$ apparent engineering strain. The second material is PLA (polylactic acid) foam, i.e. a polymeric foam based on biodegradable and biocompatible polyester (prepared by the CERM, University of Liège [7]). The specimen used ( $8.2 \mathrm{~mm}$ in diameter and $12.5 \mathrm{~mm}$ in height, with an apparent volume density of $15 \%$ ) was compressed from $0 \%$ to $42 \%$ apparent engineering strain. The last material presented is cancellous tissue of a deer antler [8]. The specimen used $(7.8 \mathrm{~mm}$ in diameter and $11.96 \mathrm{~mm}$ in height, with an apparent volume density of $18.7 \%$ ) was compressed from $0 \%$ to $4.1 \%$ apparent engineering strain.

The experimental data (Figure 1(a) to (c) - dashed lines) shows that, within the testing range, both the aluminium foam and PLA foam specimens show apparent yield behavior. However, the trabecular antler bone only shows a stiffening of an apparent linear behavior. The three specimens described earlier were each modeled as cylinders with mechanical parameters (Young's modulus, yield stress) as found in the literature for their bulk material and morphological parameters (apparent density and fabric tensor) derived from the CT data. To represent the testing boundary conditions, a displacement was applied on one side of the cylinder (vertical displacement with free in plane movement) while the other side of the cylinder was modeled to be in contact (frictionless conditions) with a rigid plane. On the contact plane, one central node of the cylinder was constrained in the horizontal plane to prevent rigid body modes. The computed external force was finally compared to the experimental one. The morphological data was initially computed as a mean over the all volume of the specimen. However, such a model allows only for the representation of the linear apparent behavior and of the apparent yield stress value (Figure 1(a) and (b) - plain black line). When extracting morphological data on smaller regions of interest (ROI), the apparent yield strain is retrieved as well (colored plain lines on the same figures). When using up to 16 divisions per specimen, one can also retrieve the early post yield behavior showing apparent softening of the specimens. For the trabecular antler bone (Figure 1(c)), increasing the morphological accuracy allows a better representation of the out-of-linearity apparent behavior. While the simulations using a large number of ROI show promising results, some effort has still to be done on the convergence rate of these simulations as the softening is no well accounted for numerically.

\section{CONCLUSIONS}

We presented a homogeneous constitutive law based on morphological data acquisition. It provides a non-linear model of the mechanical response of bone-like materials, accounting for early post-yield behavior of the structure. This constitutive model has been applied to three materials presenting bone-like morphology undergoing stepwise compression. Using this model for morphological data extracted for regions of interests small enough, one can retrieve not only the linear behavior of the structure but also the apparent yield stress and strain and early post yield softening. 


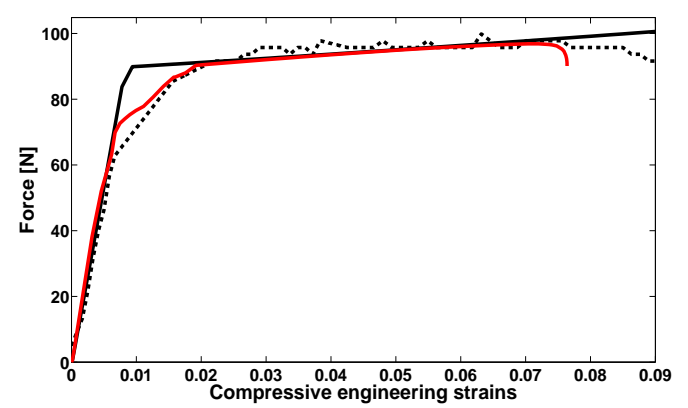

(a) Aluminium alloy

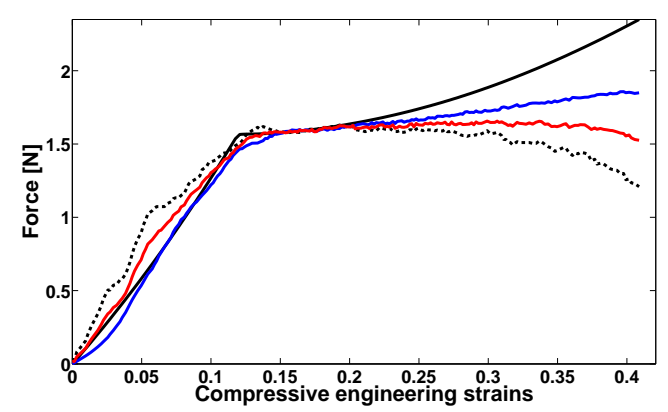

(b) PLA foam

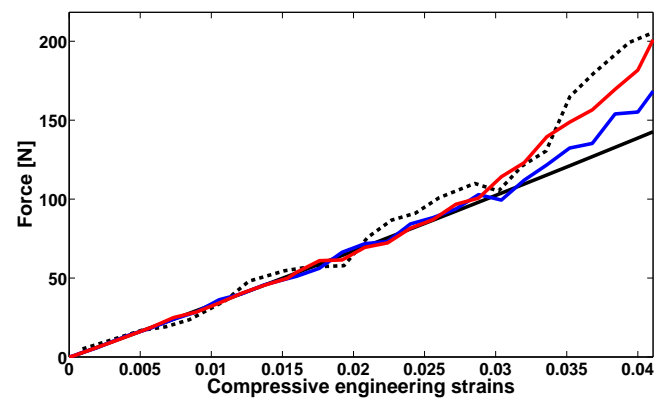

(c) Trabecular bone of deer antler

Figure 1: Computed (plain lines) and experimental (dashed lines) forces [N] vs. compressive engineering strains [-]. plain black : morphological data is computed for 1 ROI per sample ; plain blue : morphological data is computed for 8 ROI per sample ; plain red : morphological data is computed for 16 ROI per sample

\section{REFERENCES}

[1] M. Doblaré and J.M. García, Anisotropic bone remodelling model based on a continuum damage-repair theory, J. Biomech., 35, 1-17, 2002.

[2] S.C. Cowin, R.T. Hart, J.R. Balser, and D.H. Kohn, Functional adaptation in long bones: establishing in vivo values for surface remodeling rate coefficients, J. Biomech., 18, 665-684, 1985.

[3] J. Lemaitre and R. Desmorat, Engineering Damage Mechanics: Ductile, Creep, Fatigue and Brittle Failures, Springer, 2005.

[4] M. Mengoni and J.P. Ponthot, Isotropic continuum damage/repair model for alveolar bone remodeling, J. Comp. App. Math., 234, 2036-2045, 2010

[5] Metafor, A large strain finite element code, LTAS-MN2L, University of Liège, http://metafor.ltas.ulg.ac.be/ , 2010.

[6] A. Nazarian and R. Müller, Time-lapsed microstructural imaging of bone failure behavior, $J$. Biomech., 37, 55-65, 2004.

[7] V. Maquet, D. Martin, F. Scholtes, R. Franzen, J. Schoenen, G. Moonen, and R. Jérôme, Poly(D,L-lactide) foams modified by poly(ethylene oxide)-block-poly(D,L-lactide) copolymers and a-FGF : in vitro and in vivo evaluation for spinal cord regeneration, Biomaterials, 22, 11371146, 2001.

[8] Léonard, A., Guiot, L., Pirard, J. P., Crine, M., Balligand, M., and Blacher, S. Non-destructive characterization of deer antlers by X-ray microtomography coupled with image analysis, $J$. Microscopy, 225, 258263, 2007. 